\title{
Correction to: Effectiveness and Safety of Dabigatran Compared to Vitamin K Antagonists in Non-Asian Patients with Atrial Fibrillation: A Systematic Review and Meta-Analysis
}

\author{
Carlos Escobar ${ }^{1}\left(\mathbb{D} \cdot\right.$ Vivencio Barrios $^{2}$ - Gregory Y. H. Lip ${ }^{3}$. Alpesh N. Amin ${ }^{4} \cdot$ Ariadna Auladell-Rispau $^{5}$. \\ Marilina Santero ${ }^{5}$ Josefina Salazar ${ }^{5} \cdot$ Carolina Requeijo $^{5}$
}

Published online: 14 February 2022

(c) Springer Nature Switzerland AG 2022

\section{Correction to: Clinical Drug Investigation \\ https://doi.org/10.1007/s40261-021-01091-w}

The original version of this article unfortunately contained a mistake. Page 950, Table 1: row 3 in column 7-GI bleeding, which previously read $\mathrm{Y} \uparrow$ should read Limit $\uparrow$.

Corrected Table 1 is given in the following page.

The original article can be found online at https://doi.org/10.1007/ s40261-021-01091-w.

Carlos Escobar

escobar_cervantes_carlos@hotmail.com

1 Servicio de Cardiología, Hospital Universitario La Paz,

Paseo de la Castellana 261, 28046 Madrid, Spain

2 Servicio de Cardiología, Hospital Universitario Ramón y Cajal, Madrid, Spain

3 University of Liverpool and Liverpool Heart and Chest, Liverpool, UK

4 Department of Medicine, School of Medicine, University of California Irvine, Irvine, CA, USA

5 Grup de Recerca d'Epidemiologia Clínica i Serveis Sanitaris, Institut d'Investigació Biomèdica Sant Pau (IIB Sant Pau), Barcelona, Spain 


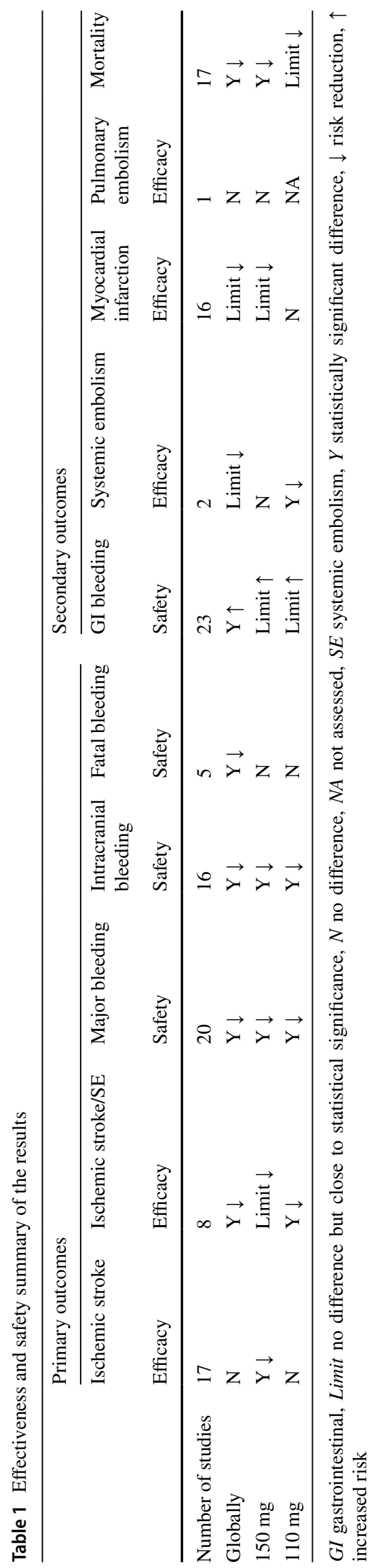

